\title{
PIGF mediates neutrophil elastase-induced airway epithelial cell apoptosis and emphysema
}

\author{
Hsin-Han Hou ${ }^{1,2}$, Shih-Lung Cheng ${ }^{3,4}$, Kuei-Pin Chung $^{5}$, Shu-Chen Wei ${ }^{6}$, Po-Nien Tsao ${ }^{6}$, Hsuan-Hsuan Lu ${ }^{1,2}$, \\ Hao-Chien Wang ${ }^{1,2^{*}+}$ and Chong-Jen $\mathrm{Yu}^{1,2^{*}+}$
}

\begin{abstract}
Background: Chronic pulmonary obstructive disease (COPD) has become the fourth leading cause of death worldwide. Cigarette smoking induces neutrophil elastase (NE) and contributes to COPD, but the detailed mechanisms involved are not fully established. In an animal model of pulmonary emphysema, there are increased expressions of placenta growth factor (PIGF) and lung epithelial (LE) cell apoptosis. This study hypothesized that excessive NE may up-regulate PIGF and that PIGF-induced LE apoptosis mediates the pathogenesis of pulmonary emphysema.
\end{abstract}

Methods: Human bronchial epithelial cells, BEAS-2B, and primary mouse type II alveolar epithelial cells were treated with NE. The PIGF promoter activity was examined by luciferase activity assay, while PIGF expression and secretion were evaluated by RT-PCR, Western blotting, and ELISA. Both cell lines were treated with PIGF to evaluate its effects and the downstream signaling pathways leading to LE cell apoptosis. PIGF knockout and wild-type mice were instilled with NE to determine the roles of PIGF and its downstream molecules in NE-promoted mice pulmonary apoptosis and emphysema phenotype.

Results: The transcriptional factor, early growth response gene-1, was involved in the NE-promoted PIGF promoter activity, and the expression and secretion of PIGF mRNA and protein in LE cells. PIGF-induced LE cell apoptosis and NE-induced mice pulmonary apoptosis and emphysema were mediated by the downstream c-Jun N-terminal kinase (JNK) and protein kinase C (PKC) $\delta$ signaling pathways.

Conclusion: The NE-PIGF-JNK/PKC $\delta$ pathway contributes to the pathogenesis of LE cell apoptosis and emphysema. PIGF and its downstream signaling molecules may be potential therapeutic targets for COPD.

Keywords: Placenta growth factor, Chronic pulmonary obstructive disease, Neutrophil elastase, Apoptosis, Emphysema

\section{Background}

Chronic pulmonary obstructive disease (COPD) is predicted to become the fourth leading cause of death worldwide by $2030[1,2]$. Due to the aging population and increasing number of smokers, the burden of medical and social resources for COPD is estimated to be US\$47 trillion by 2030 [3]. Although there are many mediators (i.e., inflammatory cells, lipids, reactivate oxygen species, nitric oxide, peptides, che-

\footnotetext{
* Correspondence: haochienwang@gmail.com; jefferycjyu@ntu.edu.tw ${ }^{\dagger}$ Equal contributors

'Departments of Internal Medicine, National Taiwan University Hospital, No. 7, Chung-Shan South Road, Taipei, Taiwan

${ }^{2}$ Department of Internal Medicine, National Taiwan University, College of Medicine, Taipei, Taiwan

Full list of author information is available at the end of the article
}

mokines, cytokines, growth factors, and proteases) and cellular pathways (e.g., inflammation, apoptosis, senescence and repair) involved in the pathogenesis of COPD, increasing evidence indicates that proteases provide vital contributions to all mediators and cellular pathways $[4,5]$. However, to date, the detailed pathogenic mechanisms of protease-mediated COPD are not fully understood $[3,6]$.

In developed countries, the major factor for the pathogenesis and progression of COPD is cigarette smoke (CS). Exposure to CS results in chronic inflammation, elevated oxidative stress, and protease-anti-protease imbalance within the respiratory system [7]. The protease-anti-protease imbalance is triggered by the infiltration of inflammatory cells like neutrophils, macrophages, and $\mathrm{CD}^{+} \mathrm{T}$ lymphocytes

\section{Biomed Central}

(c) 2014 Hou et al.; licensee BioMed Central Ltd. This is an Open Access article distributed under the terms of the Creative Commons Attribution License (http://creativecommons.org/licenses/by/4.0), which permits unrestricted use, distribution, and reproduction in any medium, provided the original work is properly credited. The Creative Commons Public Domain Dedication waiver (http://creativecommons.org/publicdomain/zero/1.0/) applies to the data made available in this article, unless otherwise stated. 
[7-11]. Proteolytic enzymes of neutrophils and macrophages, neutrophil elastase (NE), and matrix metalloproteinase (MMP)-12, degrade their respective inhibitors. Thus, the interaction promotes protease-anti-protease imbalance and destroys the pulmonary parenchyma with alveolar space dilatation, i.e. emphysema, which is a major component of COPD [12].

Neutrophil elastase is a secreted serine protease that degrades extracellular matrix like elastin, which contributes to the recoil capacity of alveoli [13]. Other than proteolytic activity, NE up-regulates elafin, interleukin-8, MUC4, and MUC5AC, and promotes the secretion of mucin in LE cells [14-18]. Excessive NE also results in LE cell apoptosis through protease-activated receptor (PAR)-1, which is abrogated by treatment with retinoic acid $[19,20]$.

Apoptosis of LE cells results in the loss of lung parenchyma and is a potential pathogenic mechanism for emphysema and COPD [21]. Placenta growth factor (PlGF) induces apoptosis of type II alveolar epithelial cells (AEC II) such that PlGF transgenic mice develop a phenotype of pulmonary emphysema [22]. PIGF is a member of the vascular endothelial growth factor family that promotes angiogenesis [23,24]. PlGF expression is abundant in the placenta, heart, lungs, thyroid, brain, and skeleton muscle during fetal development, but declines in adulthood [25]. Higher levels of PIGF have been shown in serum and broncho-alveolar lavage (BAL) fluid of COPD patients and the PIGF levels is inversely proportional to lung function deterioration [26]. Porcine pancreatic elastase (PPE), a recombinant porcine elastase for the animal model of emphysema, has also been shown to increase PlGF expression in LE cells and promote LE cells apoptosis [27]. However, the role of NE in human COPD has not been established.

Under the hypothesis that NE, like PPE, up-regulates PlGF expression and leads to LE cell apoptosis and pulmonary emphysema. This study demonstrates that the NE-promoted PlGF expression and secretion in LE cells and lungs. Early growth response gene (Egr)-1 is a transcriptional factor responsible for the up-regulation of PlGF by NE in LE cells. PlGF induces apoptosis through the c-Jun $\mathrm{N}$-terminal kinase (JNK) and protein kinase $\mathrm{C}$ $(\mathrm{PKC}) \delta$ signaling pathways. Ablation of PlGF protects mice from NE-induced pulmonary apoptosis and emphysema. Thus, NE-induced PlGF and the downstream JNK/PKC $\delta$ signaling pathways contribute to the pathogenesis of pulmonary emphysema and COPD. Both PlGF and its downstream signaling pathways may be potential therapeutic targets for COPD.

\section{Materials and methods Reagents}

Rabbit antibodies for phosphor-P38 MAPK (p-P38 MAPK), P38 MAPK, MTF-1, p-JNK and p-PKC $\delta$ were obtained from Cell Signaling Technology (Beverly, MA, USA). Antibodies for PlGF, JNK, PKC, and Egr-1, mouse and human PlGF siRNA, mouse and human PKC $\delta$ siRNA, and the corresponding scramble siRNA were purchased from Santa Cruz (Santa Cruz, CA, USA), while NE was purchased from Abcam (Cambridge, MA, USA). Trizol reagent, SuperScript III Reverse Transcriptase and Lipofectamine 2000 were obtained from Invitrogen (Carlsbad, CA, USA). Mouse antibody for beta-actin and rabbit antibody for HIF-1alpha were purchased from Genetex (Irvine, CA, USA). Human and mouse recombinant PlGF protein and an enzyme-linked immuno-sorbent assay (ELISA) kit were obtained from $\mathrm{R}$ and D Systems (Minneapolis, MN, USA).

A dual-luciferase reporter assay system was obtained from Promega (Madison, WI, USA). Hematoxylin and Eosin, Chromatin immuno-precipitation (ChIP) Assay Kit, and EZ-Zyme Chromatin Prep Kit were purchased from Merck-Millipore (Boston, MA, USA). An in situ cell Death Detection Kit and X-tremeGENE HP DNA Transfection Reagent were purchased from Roche (Mannheim, Germany). The FITC Annexin V apoptosis detection Kit I was obtained from BD Biosciences (San Jose, CA, USA). The JNK inhibitor, SP600125, was obtained from Enzo Life Science (Plymouth Meeting, PA, USA). A SuperSensitive Polymer-HRP IHC Detection System was purchased from Biogenex (Fremont, CA, USA).

\section{Animals}

This study conformed to the Guidelines for the Care and Use of Laboratory Animals published by the United States National Institutes of Health (NIH Publication No. 85-23, revised 1996). All of the animal experiments were approved by the Institutional Animal Care and Use Committee (IACUC) of the Laboratory Animal Center, College of Medicine and Public Health of National Taiwan University. Eight-week-old male C57BL/6 wild type (WT) mice were purchased from the Laboratory Animal Center, College of Medicine and College of Public Health, National Taiwan University. The PlGF knockout (KO) mice in $\mathrm{B} 6$ background were provided by Dr. Po-Nien Tsao (National Taiwan University, Taiwan).

\section{Cell culture}

Human bronchial epithelial cells, BEAS-2B (ATCC number CRL-9609), were cultured in F12 nutrient mixture (Carlsbad, CA, USA) with $0.5 \mathrm{ng} / \mathrm{ml}$ recombinant epidermal growth factor, $500 \mathrm{ng} / \mathrm{ml}$ hydrocortisone, $0.005 \mathrm{mg} / \mathrm{ml}$ insulin, $0.035 \mathrm{mg} / \mathrm{ml}$ bovine pituitary extract, $500 \mathrm{nM}$ ethanolamine, $500 \mathrm{nM}$ phosphoethanolamine, $0.01 \mathrm{mg} / \mathrm{ml}$ transferrin, $6.5 \mathrm{ng} / \mathrm{ml} \mathrm{3,} \mathrm{3',} \mathrm{5-triiodothyronine,} 500 \mathrm{ng} / \mathrm{ml}$ epinephrine, $0.1 \mathrm{ng} / \mathrm{ml}$ retinoic acid, 10\% FCS $100 \mathrm{unit} / \mathrm{ml}$ penicillin, and $100 \mu \mathrm{g} / \mathrm{ml}$ streptomycin in a humidified 95\% 
air-5\% $\mathrm{CO}_{2}$ incubator at $37^{\circ} \mathrm{C}$. Mouse primary type II alveolar epithelial cells (AEC II) and culture medium were purchased from chi scientific (Maynard, MA, USA). Primary normal human bronchial epithelial (NHBE) cells were kindly provided by Dr. Reen Wu at University of California, Davis.

\section{Plasmids}

Human genomic DNA was extracted from BEAS-2B by a Quick-gDNA MiniPrep kit (Zymo Research, CA, USA). The $2.0 \mathrm{~kb}$ human PlGF promoter region was amplified from human genomic DNA using polymerase chain reaction (PCR) performed with $\mathrm{Hi}$ Fi Taq DNA polymerase (Geneaid, Taipei, Taiwan) as follows: 2 minutes at $94^{\circ} \mathrm{C}$, then $15 \mathrm{sec}$ at $94^{\circ} \mathrm{C}, 30 \mathrm{sec}$ at $59^{\circ} \mathrm{C}$, and $2 \mathrm{~min}$ and $30 \mathrm{sec}$ at $72^{\circ} \mathrm{C}$ for 35 cycles. The primers for $2.0 \mathrm{~kb}$ human PIGF promoter region were 5'-GCG GTAC CCA AAC TCA TAC ACA ATA GAC-3' (forward primer; italic, KpnI site) and 5'-TTA AGCT TCC GTA GGT AAG GCT GTG GCT-3' (reverse primer; italic, HindIII site). The amplified DNA fragments were cloned into pGL3 vector (Promega, WI, USA) and the sequences were confirmed by DNA sequence analysis. The pGL3 with mouse PlGF promoter was as previously described [27].

\section{Enzyme-linked immuno-sorbent assay (ELISA)}

Cellular medium from BEAS-2B and AEC II, and BAL fluid from mice were analyzed by a PlGF ELISA kit ( $R$ \& D, MN, USA) according to the manufacturer's instructions.

\section{Luciferase reporter assay}

The BEAS-2B and AEC II were co-transfected with the pGL3-PlGF promoter and pRenilla for $24 \mathrm{~h}$ via Lipofectamine 2000 and X-tremeGENE HP DNA Transfection Reagent, and then collected and analyzed on a dualluciferase reporter assay system (Promega, WI, USA) using a lumicounter Packard BL10000 according to the manufacturer's instructions.

\section{Protein extraction and immuno-blot analysis}

The BEAS-2B and AEC II were lysed using RIPA lysis buffer (Genestar, Taipei, Taiwan), containing 1\% NP-40, $0.1 \%$ SDS, $150 \mathrm{mM}$ sodium chloride, $0.5 \%$ sodium deoxycholate, and $50 \mathrm{mM}$ Tris with a protease inhibitor cocktail (Bionovas, Toronto, Canada) and PhosSTOP (Roche, Basilea, Switzerland). The cell lysates were centrifuged at $12,000 \mathrm{rpm}$ for $5 \mathrm{~min}$ and the resulting supernatant was collected.

The extracted protein was quantified by protein assay. Equal amounts of protein were separated using 10\% SDS-polyacrylamide gel electrophoresis and transferred to Immobilon-P membranes (Millipore, MA, USA). After blocking with $5 \%$ skimmed milk, the membranes were incubated with various primary antibodies and then incubated with the corresponding secondary antibodies. The protein bands were detected using an Immobilon Western Chemi-luminescent HRP Substrate (Millipore, MA, USA) and quantified by the ImageQuant 5.2 software (Healthcare Bio-Sciences, PA, USA).

\section{Terminal deoxynucleotidyl transferase dUTP nick end labeling (TUNEL) assay}

The BEAS-2B and AEC II, and OCT-embedded lung tissue from the mice were analyzed for the apoptosis level using an in situ cell Death Detection Kit (Roche, Basilea, Switzerland) according to the manufacturer's instructions. Fluorescence-positive cells were photographed by a Leica DM 4000B microscope (Leica, Solms, Germany).

\section{Flow cytometry analysis}

The BEAS-2B and AEC II were analyzed on a FITC Annexin V apoptosis detection Kit I (Franklin Lakes, NJ, USA) according to the manufacturer's instructions. The FITC-positive cells were analyzed using a FACS Calibur flow cytometer (Becton Drive, NJ, USA).

\section{Immuno-histochemistry (IHC) assay}

Paraffin was removed from paraffin-embedded tissue sections by xylene, dehydrated by ethanol, and re-hydrated by PBS. After treatment with $3 \% \mathrm{H}_{2} \mathrm{O}_{2}$, the sections were applied to a SuperSensitive Polymer-HRP IHC Detection System (Biogenex, CA, USA) and incubated with PlGF, p-JNK, and p-PKC $\delta$ antibodies as primary antibodies. The stained-sections were photographed using a Leica DM 4000B microscope (Leica, Solms, Germany).

\section{Hematoxylin and eosin ( $\mathrm{H}$ and $\mathrm{E})$ staining}

Paraffin was removed from paraffin-embedded tissue sections by xylene, dehydrated by ethanol, and re-hydrated by PBS. Sections stained with $\mathrm{H}$ and $\mathrm{E}$ were photographed by a Leica DM 4,000 B microscope (Leica, Solms, German).

\section{NE-induced emphysema}

The dose of NE was four-fold higher than that of porcine pancreatic elastase according to previous report [28] and the methodology of intra-tracheal instilling NE was performed as previously described [29]. Briefly, eight-week-old mice were intra-tracheally given saline $(\mathrm{CON}), 400 \mathrm{mU} / \mathrm{ml}$ $\mathrm{NE}(\mathrm{NE}), 400 \mathrm{mU} / \mathrm{ml} \mathrm{NE}$ with $50 \mathrm{mg} / \mathrm{kg}$ JNK inhibitor SP600125 (NE SP), $3 \mathrm{mg} / \mathrm{kg}$ scramble siRNA (NE Si-Sc), $3 \mathrm{mg} / \mathrm{kg}$ mouse PKC siRNA (NE Si-PK) and $3 \mathrm{mg} / \mathrm{kg}$ PIGF siRNA (NE Si-Pl) weekly for one month. The dose of siRNA instillation was according to a previous study $[27,30]$. Each experimental group had five mice and the processing of lung tissues and BAL fluid were performed as previously described [27,29]. 


\section{Reverse-transcriptional (RT)-PCR assay}

Total RNA of BEAS-2B and AEC II were extracted by Trizol Reagent (Invitrogen, CA, USA) according to the manufacturer's instructions. Total RNA $(5 \mu \mathrm{g})$ was used in the RT reactions using a SuperScript III Reverse Transcriptase kit (Invitrogen, CA, USA) according to the manufacturer's instructions to synthesize the cDNA. The human PIGF and glyceraldehyde 3-phosphate dehydrogenase (GAPDH) cDNA fragments were amplified from the cDNA by PCR, performed with Dream Taq DNA polymerase (Fermentas, MA, USA) as follows: $5 \mathrm{~min}$ at $95^{\circ} \mathrm{C}$, then $30 \mathrm{sec}$ at $98^{\circ} \mathrm{C}, 30 \mathrm{sec}$ at $59^{\circ} \mathrm{C}$, and $1 \mathrm{~min}$ at $72^{\circ} \mathrm{C}$ for 35 cycles. The primers for $164 \mathrm{bp}$ human PlGF cDNA fragment were $5^{\prime}$-GGC GAT GAG
AAT CTG CAC TGT-3' and 5'-GAA GAT GAA GCC GGA AAG GTG-3'. The primers for 530 bp human GAPDH cDNA fragment were 5'-GGG CGC CTG GTC ACC AGG GCT G-3' and 5'-GGG GCC ATC CAC AGT CTT CTG-3'. The primer sets for mouse PlGF and GAPDH was as previously described [27].

\section{Chromatin immuno-precipitation (ChIP)}

Genomic DNA fragment from BEAS-2B were prepared by the EZ-Zyme Chromatin Prep Kit (Millipore, MA, USA) and analyzed using the Chromatin immunoprecipitation (ChIP) Assay Kit (Millipore, MA, USA) to evaluate the associated levels of Egr-1 and PlGF promoter regions. The antibody of Egr-1 was used for
(A)

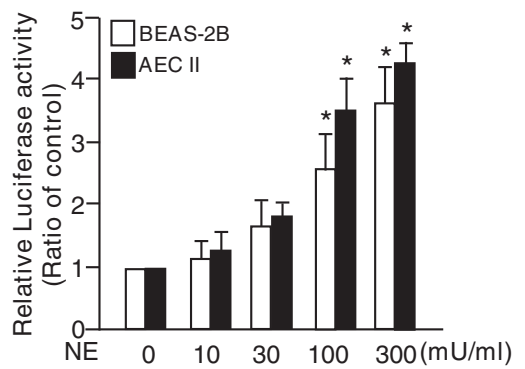

(C)
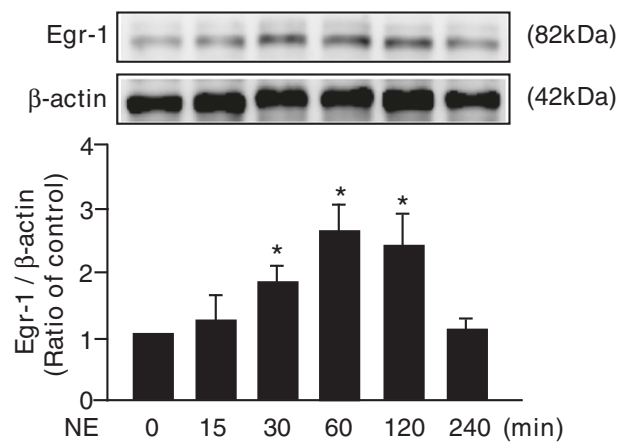

(E) Scramble siRnA

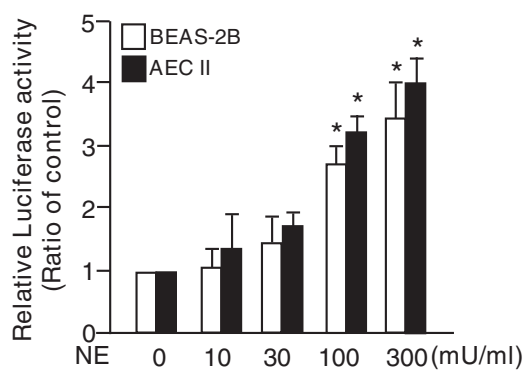

(B)

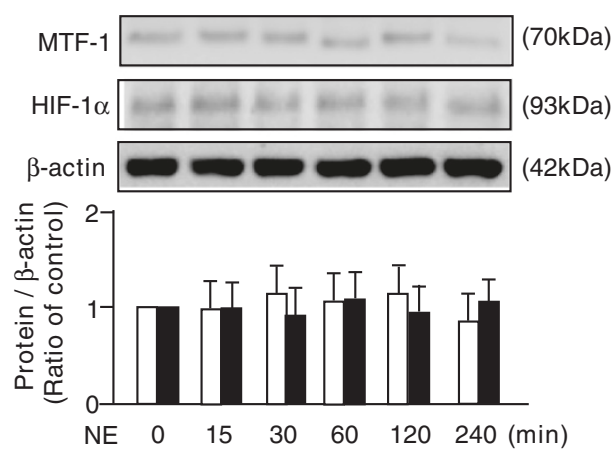

(D)

(F) Egr-1 siRNA
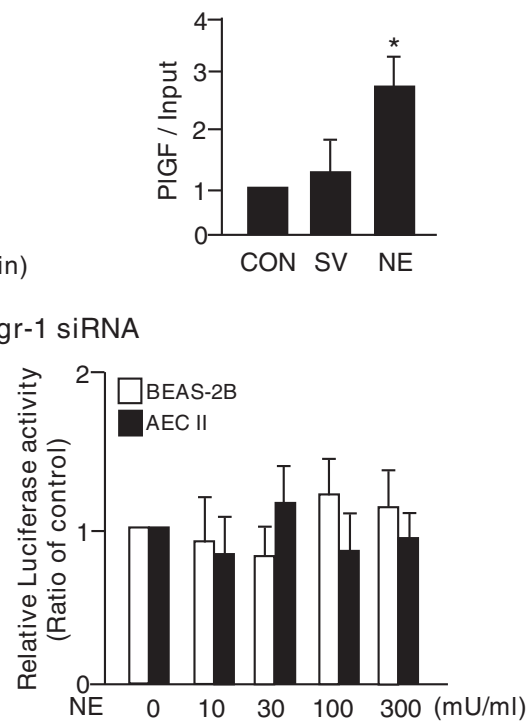

Figure 1 NE-induced PIGF promoter activity is mediated by Egr-1. (A, E, and F) The placenta growth factor (PIGF) promoter activity was evaluated by luciferase activity. (B) Hypoxia inducible factor (HIF)-1alpha and metal-regulatory transcription factor (MTF)-1, (C) Egr-1, and $\beta$-actin were analyzed by Western blot analysis. (D) The association of Egr-1 and PIGF promoter fragment was evaluated by chromatin immuno-precipitation assay. Data are presented as mean \pm SEM. ${ }^{*} p<0.05$ vs. vehicle-treated group. 


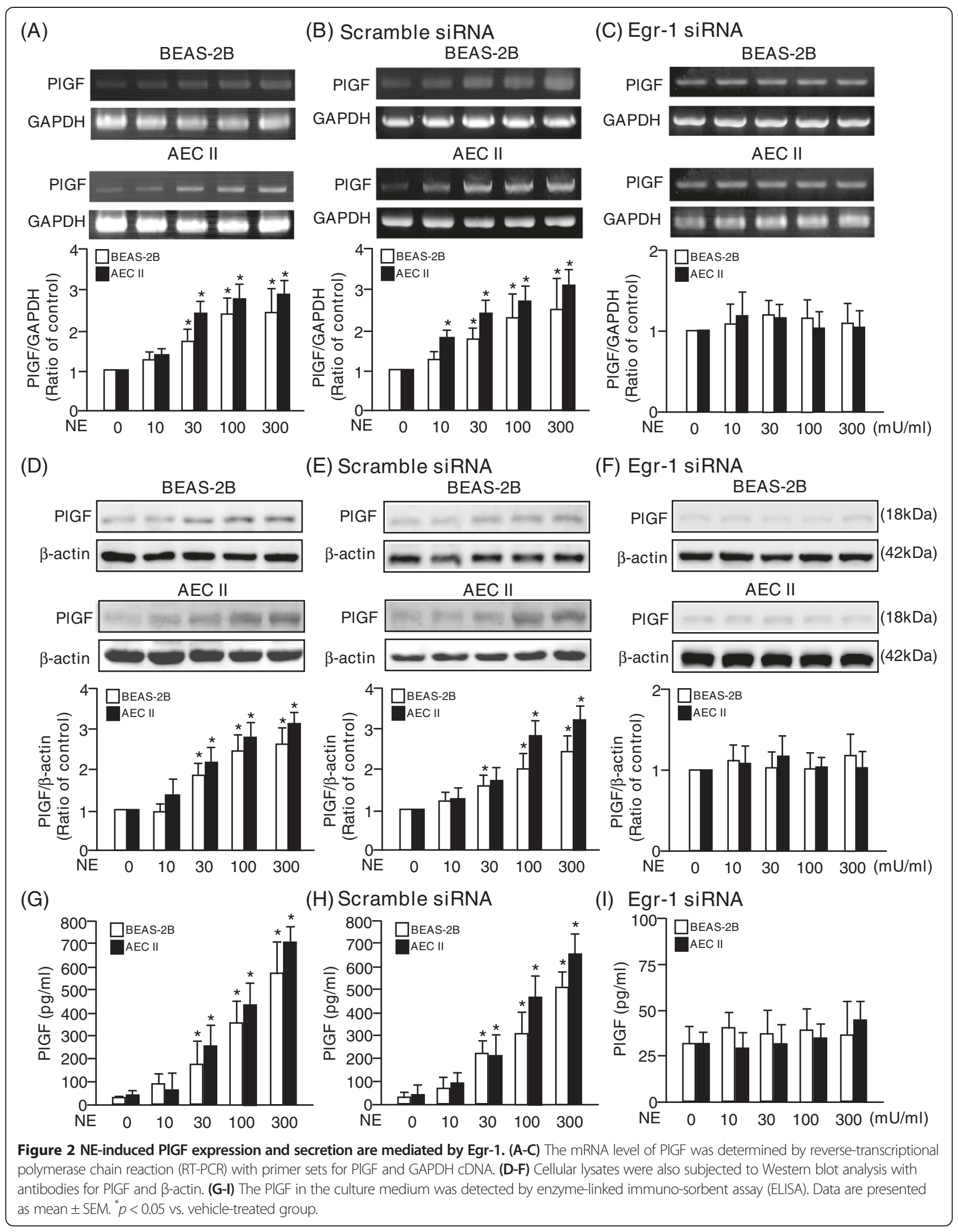




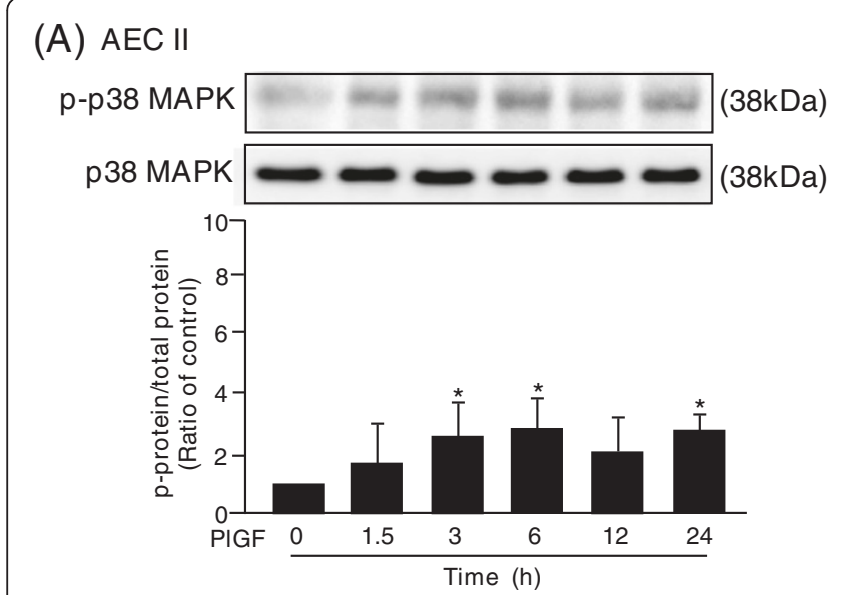

(C) AEC ॥
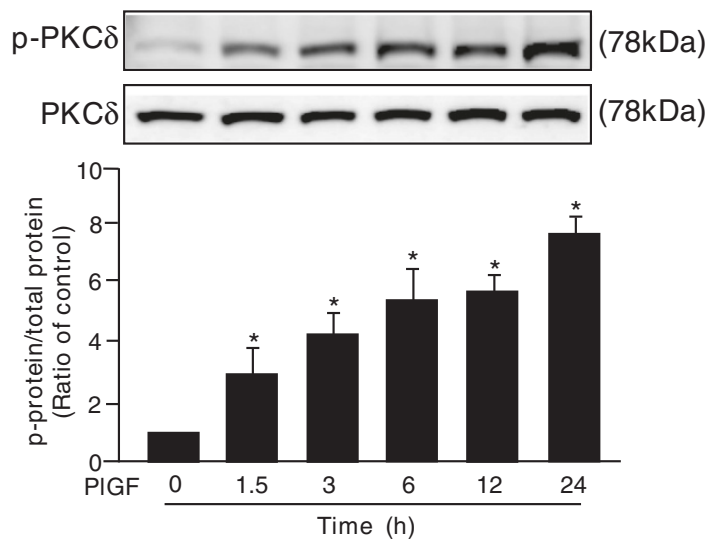

(B) AEC II

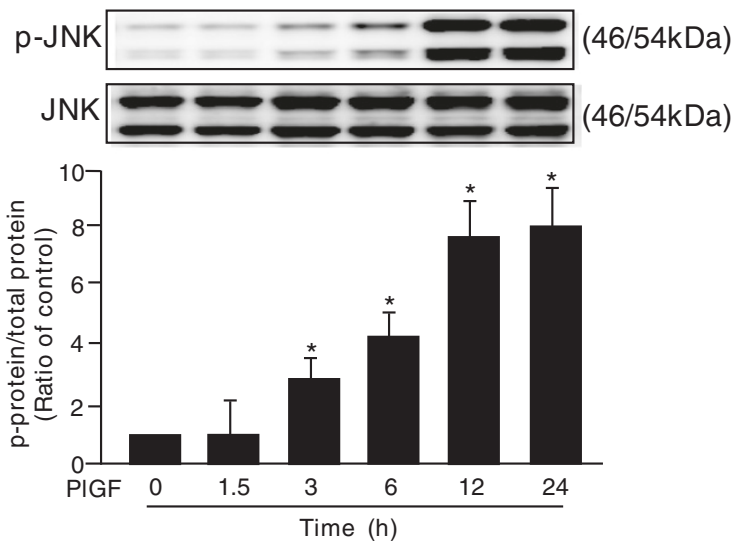

(D) BEAS-2B

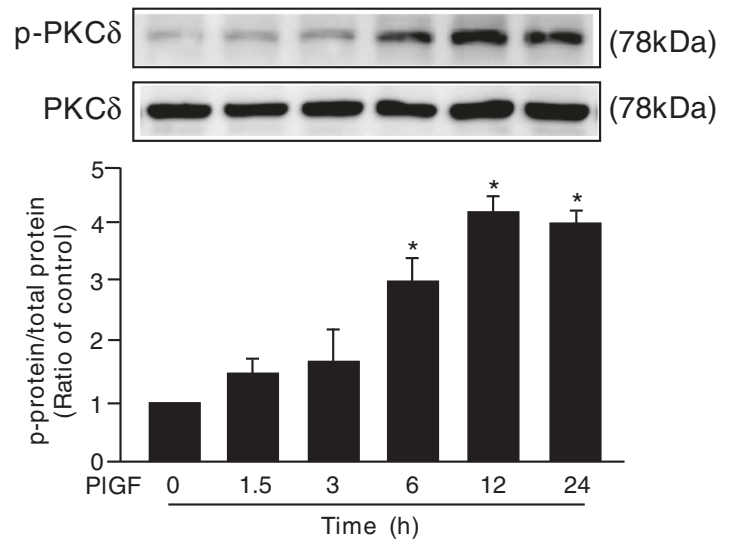

Figure 3 PIGF activates JNK and PKC $\delta$ signaling pathways in LE cells. (A-C) AEC II and (D) BEAS-2B were treated with 100 ng/ml recombinant human mouse PIGF respectively for 0-24 h. Cellular lysates were subjected to Western blot analysis with antibodies for phosphorylated p38 MAPK (p-p38 MAPK) and p38 MAPK (A), phosphorylated JNK (p-JNK) and JNK (B), phosphorylated PKC $\delta$ (p-PKC $)$ and PKC $(\mathbf{C}$ and D). Data are presented as mean \pm SEM. ${ }^{*} p<0.05$ vs. vehicle-treated group.

immuno-precipitation and the primer set $\left(5^{\prime}\right.$-CAC TTT CCA AGA ATG CCT ATG TCC ATT C-3' and 5' -TTA AGC TTC CGT AGG TAA GGC TGT GGC T-3') were used to amplify the human PlGF promoter fragment according to the manufacturer's instructions.

\section{Statistical analysis}

The results were presented as mean \pm SEM from five independent experiments and animals. The Mann-Whitney test was used to compare two independent groups. Kruskal-Wallis with Bonferroni post hoc analysis was used for multiple testing. Statistical analyses were performed using the SPSS version 8.0 (SPSS Inc., IL, USA). Statistical significance was set at $p<0.05$.

\section{Results}

NE increased PIGF promoter activity by Egr-1 in LE Cells The results revealed that treatment with $100-300 \mathrm{mU} / \mathrm{ml}$ NE for $24 \mathrm{~h}$ significantly increased PIGF promoter activity dose-dependently in human bronchial epithelial cells, BEAS-2B, and primary mouse type II alveolar epithelial cell (AEC II) (Figure 1A). Previous studies indicated that several conserved metal response elements (MRE) and hypoxia response elements (HRE) reside in mouse or human PlGF promoter regions [31,32]. However, treatment with $300 \mathrm{mU} / \mathrm{ml} \mathrm{NE}$ did not alter the expression of mental-regulatory transcription factor (MTF)-1 and hypoxia inducible factor (HIF)- $1 \alpha$ (Figure 1B).

There was a conserved Egr-1 response element in the human and mouse PlGF promoter regions near the transcriptional start site [32,33]. Western blotting revealed that $300 \mathrm{mU} / \mathrm{ml} \mathrm{NE}$ challenge transiently increased Egr-1 expression in BEAS-2B (Figure 1C). By ChIP, treatment of $300 \mathrm{mU} / \mathrm{ml} \mathrm{NE}$ for $1 \mathrm{~h}$ triggered the binding of Egr-1 and PlGF promoter fragments in BEAS-2B (Figure 1D) and pre-treatment with Egr-1 siRNA inhibited the NE-increased PlGF promoter 


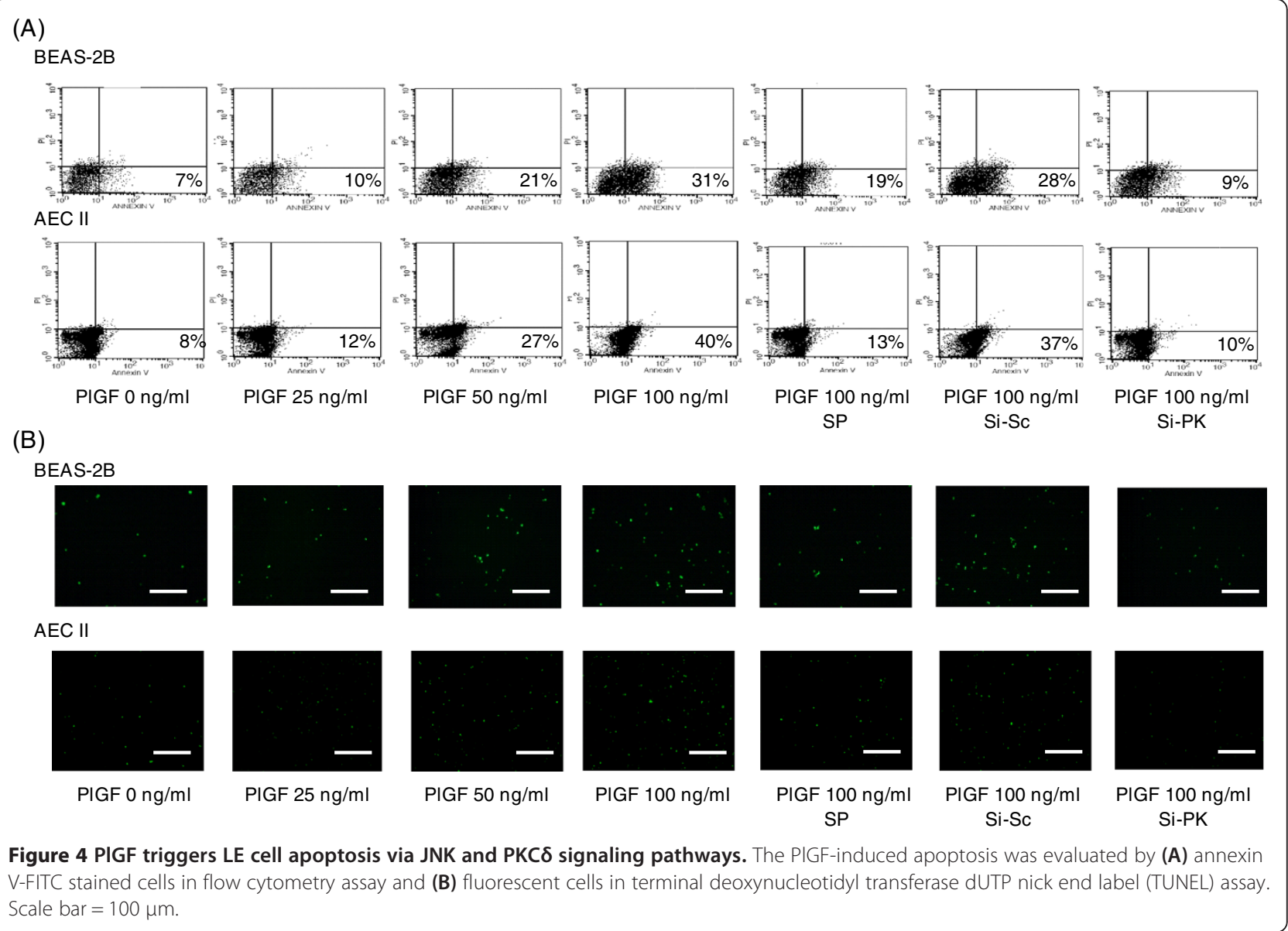

activity in BEAS-2B and AEC II (Figures $1 \mathrm{E}$ and F). Thus, NE increased PlGF promoter activity through the association of Egr-1 and the PlGF promoter fragment.

\section{NE increased PIGF expression in LE Cells}

$\mathrm{NE}(100 \mathrm{mU} / \mathrm{ml})$ had been reported to up-regulate elafin expression in A549 cells [14] and PlGF was majorly secreted by AEC II $[22,34]$. To test whether NE could induce PIGF expression, BEAS-2B and AECII were treated with of $0-300 \mathrm{mU} / \mathrm{ml} \mathrm{NE}$ for $24 \mathrm{~h}$. PlGF mRNA and protein level were increased after NE challenge in a dose-dependent manner and Egr-1 siRNA pretreatment abrogated the NE-induced PIGF mRNA (Figure 2A-C) and protein (Figure 2D-F) expressions in BEAS-2B and AEC II. Moreover, Egr-1 siRNA also blocked the NE-induced PIGF secretion in medium of BEAS-2B and AEC II (Figure 2G-I).

Moreover, NE increased the PlGF expression in endothelial cell but not in fibroblast cell (Additional file 1 and Additional file 2: Figures S1A and S1B). Taken together, other than natural activity of proteolysis, NE increased the PIGF expressions and promoted PlGF secretion.
PIGF induced apoptosis in LE Cells via JNK and PKC $\delta$ signaling pathways

A previous study indicated that $100 \mathrm{ng} / \mathrm{ml}$ PlGF induced MLE-15 cell apoptosis with an unknown mechanism [22]. It has been previously demonstrated that PIGF increased apoptosis in MLE-15 cells and BEAS$2 \mathrm{~B}$ via JNK and $\mathrm{p} 38$ mitogen-activated protein kinase (MAPK) signaling pathways $[27,35]$. In order to confirm and explore the mechanisms underlying PlGFinduced LE cells apoptosis, BEAS-2B and AEC II were treated with $100 \mathrm{ng} / \mathrm{ml}$ recombinant PlGF for $24 \mathrm{~h}$.

Although the results of Western blot analysis revealed that PlGF didn't activate p38 MAPK significantly, PlGF induced a prolonged and enhanced phosphorylation of JNK and PKCS in AEC II (Figure 3A-C). PIGF also activated PKC $\delta$ pathways in BEAS-2B (Figure 3D). Blockade of JNK or PKC $\delta$ signaling by JNK inhibitor, SP600125, or transfection with PKC $\delta$ siRNA had no effect on PlGF-activated PKC $\delta$ or JNK (Additional file 3: Figure S2), suggesting no crosstalk between PlGF-activated JNK and PKC $\delta$ signaling pathways.

Further evaluating the roles of JNK and $\mathrm{PKC} \delta$ in PIGF-induced apoptosis, BEAS-2B and AEC II were pretreated with JNK inhibitor or transfected with PKC $\delta$ 
siRNA to block the PlGF down-stream signaling pathways, then treated with $0-100 \mathrm{ng} / \mathrm{ml}$ PlGF for $24 \mathrm{~h}$. Results of flow cytometry assay (Figure 4A) and TUNEL assay (Figure $4 \mathrm{~B}$ ) indicated that first, exogenous PlGF dose-dependently increased BEAS-2B and AEC II apoptotic levels and second, the JNK and PKC $\delta$ signaling pathways played crucial roles in PlGF-stimulated LE cell apoptosis.

The impact of NE-induced endogenous PlGF on NEinduced LE cell apoptosis was further evaluated in normal human bronchial epithelial cells (NHBE) with serum-free medium, which was the applicable condition for NE-digestion. This study also further proved that NE caused NHBE apoptosis and blocked endogenous PIGF signaling by VEGFR1 neutralized antibody, which attenuated the NE-induced NHBE apoptosis and NEactivated JNK and PKC $\delta$ signaling pathways (Additional file 1 and Additional file 4: Figure S3).
Intra-tracheal instillation of NE increased PIGF expression and secretion and activated downstream JNK and PKC $\delta$ signaling pathways

The role of PlGF in NE-induced LE cells apoptosis and emphysema was further confirmed in an animal model. Wild-type (C57BL/6) and PlGF $\mathrm{KO}$ mice were intratracheally treated with saline $(\mathrm{CON})$ or $400 \mathrm{mU} / \mathrm{ml} \mathrm{NE}$ (NE) weekly for one month. The pathology of the NEtreated mice showed elevated PlGF expression in alveolar epithelial cell (Figure 5A) and adjacent endothelial cells than controls (Additional file 2: Figure S1C). Moreover, NE-treated mice displayed more phosphorylated JNK and PKC $\delta$ levels than the control mice (Figure 5A).

In contrast, ablation of PlGF limited the expression of PIGF and blocked the NE instillation-induced activation of JNK and PKC (Figure 5B). The BAL fluid from NEtreated mice also had higher PIGF levels compared to the control mice. However, there was a lack of PIGF in

(A) WT mice
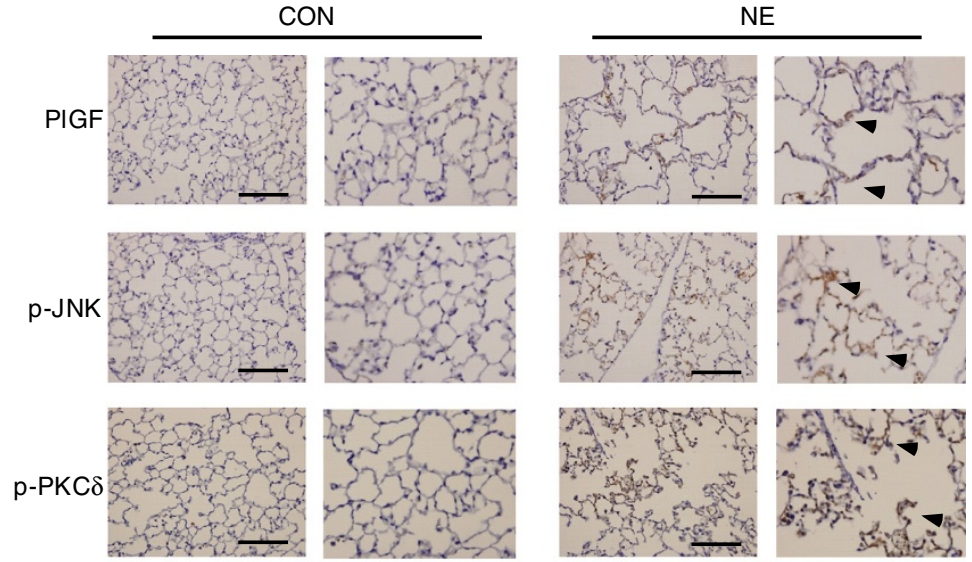

(B) PIGF KO mice

CON
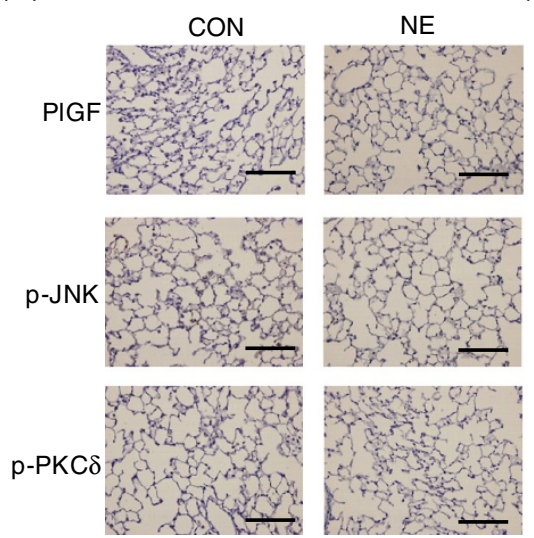

(C)

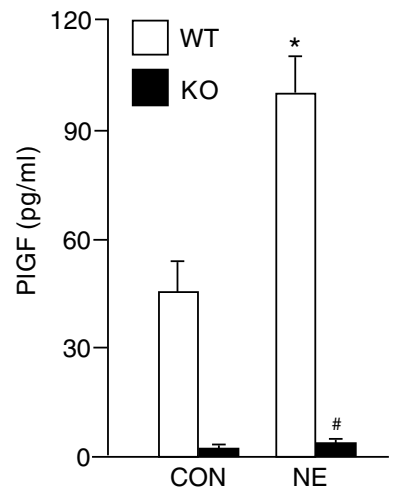

Figure 5 NE increases expression of PIGF and activation of JNK and PKC $\delta$ signaling. (A and B) Paraffin-embedded lung tissue sections were used for immuno-histochemistry $(\mathrm{IHC})$ analysis and incubated with antibodies of PIGF, p-JNK and p-PKC $\delta$. Arrow heads indicated positive stain of PIGF, p-JNK and p-PKC $\delta$ in LE cells. (C) Mice broncho-alveolar lavage fluid was analyzed for the PIGF level by ELISA. Scale bar $=200 \mu \mathrm{m}$. Data are presented as mean \pm SEM. ${ }^{*} p<0.05$ vs. vehicle-treated group; $\# p<0.05$ vs. corresponding WT group. 
KO mice (Figure 5C). These results demonstrated that $\mathrm{NE}$ instillation increased the expression and secretion of PlGF, as well as the activation of JNK and PKC $\delta$ in pulmonary cells.

\section{PIGF and PIGF-activated JNK and PKC $\delta$ pathways were} involved in NE-induced apoptosis and emphysema in mice

To evaluate the roles of PIGF and JNK/PKC $\delta$ signaling in NE-induced apoptosis and emphysema in an animal model, $50 \mathrm{mg} / \mathrm{kg}$ of SP600125, $3 \mathrm{mg} / \mathrm{kg}$ scramble siRNA, $3 \mathrm{mg} / \mathrm{kg}$ PKCס siRNA, or $3 \mathrm{mg} / \mathrm{kg}$ PlGF siRNA were co-treated with NE installation (NE SP, NE Si-Sc, NE Si-PK, or NE Si-Pl) on WT and PIGF KO mice weekly for one month. TUNEL assay indicated more abundant apoptotic cells in the pulmonary tissue of NE-treated mice than control mice (Figures 6A and E). In contrast, the ablation of PlGF protected mice from NE-induced pulmonary cell apoptosis (Figure 6C and $\mathrm{E}$ ).
Moreover, NE-treated mice had the emphysema phenotype with enlargement of the alveolar space (Figure 6B), as evaluated by the mean linear intercept (MLI) (Figure 6F). On the other hand, ablation of PlGF protected mice from NE-induced pulmonary destruction (Figure 6D and F). Furthermore, blocking the JNK and PKC $\delta$ signaling pathways (NE SP and NE Si-PK) and silencing of PlGF (NE Si-Pl) abrogated the levels of NE-induced pulmonary apoptosis (Figure 6A and E) and attenuated the airspace enlargement in mice (Figure $6 \mathrm{~B}$ and F). Thus, the animal model of elastase-instillation further confirmed that the NEincreased pulmonary PlGF and the PlGF-activated JNK/ PKC $\delta$ signaling pathways were involved in NE-induced pulmonary apoptosis and emphysema in vivo.

\section{Discussion}

There are several conserved trans-elements within the human and mouse PlGF promoter regions, including MRE and HRE [31,32]. Treatment with PlGF does not

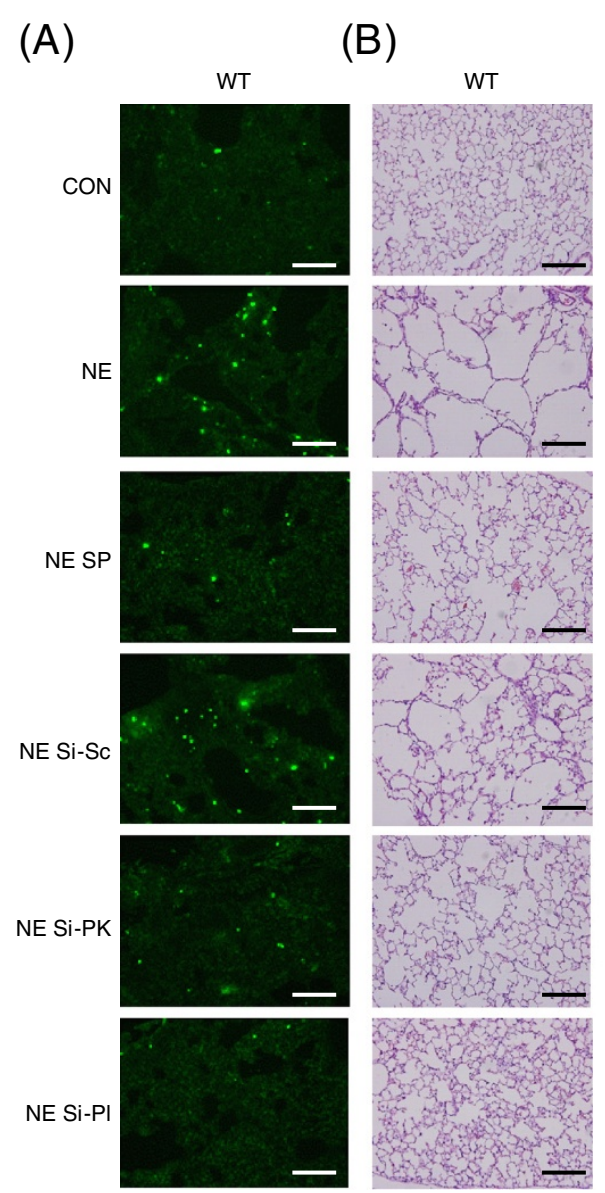

(C)

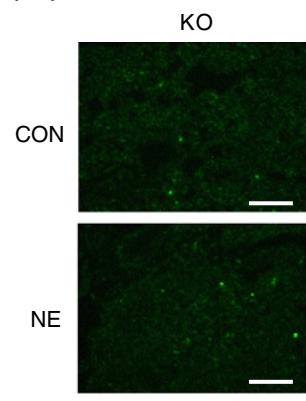

(D)

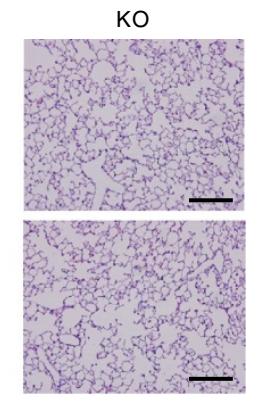

$(\mathrm{E})$

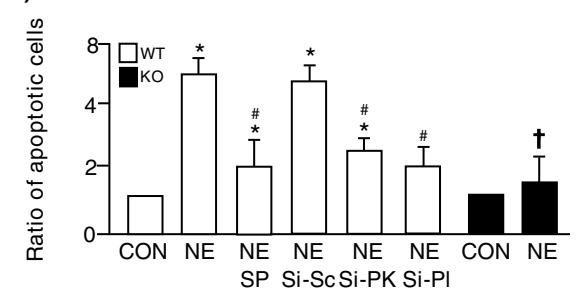

$(\mathrm{F})$

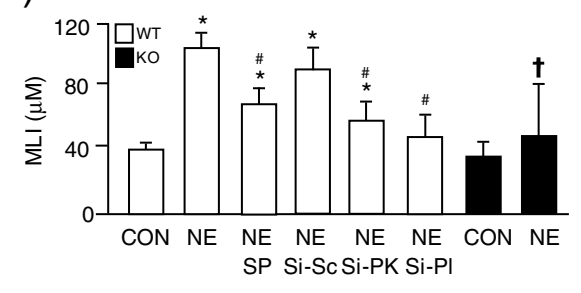

Figure 6 NE-induced pulmonary apoptosis and emphysema are mediated by PIGF and the downstream JNK/PKC $\delta$ signaling pathways. ( $A$ and C) The frozen lung tissue sections were used for TUNEL assay and (B and D) paraffin-embedded lung tissue sections were used for $H$ and E staining. (E) Apoptotic cells in TUNEL assay were quantified and (F) the mean linear intercepts (MLI) from five independent sections were evaluated. Scale bar $=200 \mu \mathrm{m}$. Data are presented as mean \pm SEM. ${ }^{*} p<0.05$ vs. vehicle-treated group; \#p $<0.05$ vs. NE-treated group; $t p<0.05$ vs. corresponding WT group. 
affect the expressions of MTF- 1 and HIF- $1 \alpha$, which are the binding proteins for MRE and HRE. A conserved Egr-1 response element (CCCCGCCCC) [36] is observed near the transcriptional start site in both mouse and human PlGF promoter. Egr-1 is a rapid response transcription factor for UV and cigarette smoke stimuli that up-regulates several genes, including PTEN, microtubuleassociated protein-1 light chain 3, and PAR-1 in LE cells [36-39]. The Egr-1-upregulated down-stream genes mediate various cellular functions like cell growth, proliferation, differentiation, and apoptosis [39]. Egr-1 also has an impact on the pathogenesis of acute lung injury [40]. A previous study has demonstrated that NE inhibitors decrease ventilator-induced Egr-1 expression [41]. In the present study, NE promotes the transient expression of Egr-1, which is involved in NE-induced PlGF expression.

The present study demonstrates that NE-induced PIGF promotes LE cell apoptosis, which corroborate the results of a previous study [22]. However, unlike previously established mechanisms of NE-induced LE cell apoptosis $[19,20]$, this study is the first to show that NE-induces LE cell apoptosis through PlGF and PlGF-mediated downstream JNK and PKC $\delta$ signaling pathways. The results of NHBE cells further indicate that NE-promoted endogenous PIGF contributes to LE cell apoptosis. Furthermore, NE up-regulates PIGF in endothelial cells and in LE cells. The PlGF-induced LE cell apoptosis may work through both autocrine and paracrine mechanism. In addition, it is interesting to know that the upregulation of PlGF is identified in an ovalbumin-induced asthma mice model wherein PlGF promotes neutrophilic chemotaxis [42]. Therefore, the positive feedback loop between NE and PIGF in the pathogenesis of COPD warrants further investigation.

Because of frequently ignored early symptoms and irreversible pulmonary damage, COPD remains a major cause of death worldwide [2]. As a chronic disease with insidious pathogenesis, COPD is difficult to diagnose early. Useful diagnostic markers will help in the early diagnosis, early treatment, and reduction of mortality and morbidity. A previous report indicates that the NE-digested product, $A \alpha-V a l 360$, may be a marker for COPD [43]. However, endogenous elastin fragments can disturb the utility of A $\alpha-\mathrm{Val} 360$ for predicting COPD.

The present study demonstrates that PlGF, which physiologically appears only in the embryonic stage, may be a suitable candidate as a diagnostic marker of early COPD. Based on the $\mathrm{IHC}$ results and BAL data in a previous study [26], COPD patients secrete and express more PlGF compared to non-COPD controls. Other than COPD, the up-regulation of PIGF is also associated with higher risk of several human diseases, including age-related macular degradation, sickle cell disease, and most kinds of tumors [24]. As PlGF expression is barely detectable in healthy adults, further investigation regarding the association between PlGF and COPD may therefore support PlGF as a candidate marker for early COPD.

A previous study indicates that mouse PlGF activates p38 MAPK and JNK signaling pathway in mouse alveolar epithelial cells, and that MLE-15 and human PIGF activates the p38 MAPK and JNK signaling pathway in BEAS-2B. In the present study, PlGF promotes only JNK and PKC $\delta$ in AEC II cell. The difference in cell systems may explain why PIGF acts through different down-stream signaling pathways. However, the JNK, p38 MAPK, and PKC $\delta$ signaling pathways should all be considered as potential therapeutic targets aside from PIGF for COPD therapy [44-46].

\section{Conclusions}

Using human and mouse LE cells as well as an in vivo model, this study demonstrates that NE challenge stimulates PlGF expression and secretion, and that PlGF promotes LE cell apoptosis via the JNK and PKC $\delta$ signaling pathways. Thus, PIGF and the downstream JNK/PKC $\delta$ signaling pathways participate in the pathogenesis of CS-related COPD and should be considered potential therapeutic targets for COPD therapy.

\section{Additional files}

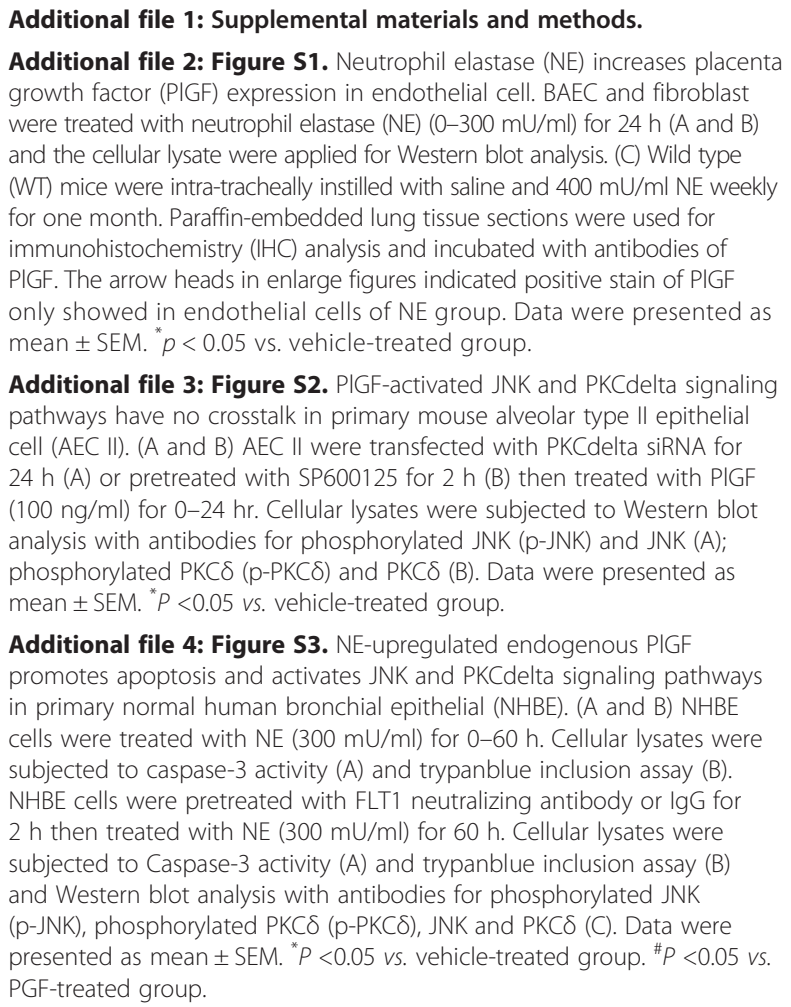

Additional file 3: Figure S2. PIGF-activated JNK and PKCdelta signaling pathways have no crosstalk in primary mouse alveolar type II epithelial cell $(A E C \|)$. (A and B) AEC II were transfected with PKCdelta siRNA for $24 \mathrm{~h}(\mathrm{~A})$ or pretreated with SP600125 for $2 \mathrm{~h}$ (B) then treated with PIGF (100 ng/ml) for 0-24 hr. Cellular lysates were subjected to Western blot analysis with antibodies for phosphorylated JNK ( $p$-JNK) and JNK (A); phosphorylated PKC $\delta$ (p-PKC $\delta$ ) and PKC $\delta$ (B). Data were presented as mean \pm SEM. ${ }^{*} P<0.05$ vs. vehicle-treated group.

Additional file 4: Figure S3. NE-upregulated endogenous PIGF promotes apoptosis and activates JNK and PKCdelta signaling pathways in primary normal human bronchial epithelial (NHBE). (A and B) NHBE cells were treated with NE $(300 \mathrm{mU} / \mathrm{ml})$ for 0-60 h. Cellular lysates were subjected to caspase-3 activity (A) and trypanblue inclusion assay (B). NHBE cells were pretreated with FLT1 neutralizing antibody or IgG for $2 \mathrm{~h}$ then treated with NE $(300 \mathrm{mU} / \mathrm{ml})$ for $60 \mathrm{~h}$. Cellular lysates were subjected to Caspase-3 activity (A) and trypanblue inclusion assay (B) and Western blot analysis with antibodies for phosphorylated JNK ( $p-J N K)$, phosphorylated PKC $\delta$ (p-PKC $\delta)$, JNK and PKC $\delta(C)$. Data were presented as mean \pm SEM. ${ }^{*} P<0.05$ vs. vehicle-treated group. ${ }^{\#} P<0.05$ vs. PGF-treated group

\section{Abbreviations}

COPD: Chronic pulmonary obstructive disease; CS: Cigarette smoke; NE: Neutrophil elastase; MMP: Matrix metalloprotease; LE: Lung epithelial; 
PIGF: Placenta growth factor; AEC II: Type II alveolar epithelial cell; MAPK: Mitogen-activated protein kinase; BAL: Broncho-alveolar lavage; WT: Wild-type; Egr-1: Early growth response gene-1; JNK: c-Jun N-terminal kinase; PKC: Protein kinase C; MTF: Mental-regulatory transcription factor; HIF: Hypoxia inducible factor; ELISA: Enzyme-linked immuno-sorbent assay; ChIP: Chromatin immuno-precipitation; NHBE: Normal human bronchial epithelial cells; KO: Knockout; TUNEL: Terminal deoxynucleotidyl transferase dUTP nick end labeling; IHC: Immuno-histochemistry; MRE: Metal response element; HRE: Hypoxia response element; PAR: Protease-activated receptor.

\section{Competing interests}

The authors declare that they have no competing interests.

\section{Author' contributions}

$\mathrm{HHH}, \mathrm{SLC}, \mathrm{HCW}$ and CJY designed research. HHH, SLC, KPC and HHL conducted experiments. SCW and PNT provided PIGF KO mice. SLC, HCW and $\mathrm{CJY}$ provided help with data interpretation. $\mathrm{HHH}$ and $\mathrm{CJY}$ wrote the paper. All authors read and approved the final manuscript.

\section{Acknowledgements}

This study was supported by grants from the National Health Research Institute (NHRI-EX101-10150SI) and Far Eastern Memorial Hospital (FEMH-2013-C-109), Taiwan.

\section{Author details}

'Departments of Internal Medicine, National Taiwan University Hospital, No. 7, Chung-Shan South Road, Taipei, Taiwan. ${ }^{2}$ Department of Internal Medicine, National Taiwan University, College of Medicine, Taipei, Taiwan. ${ }^{3}$ Department of Internal Medicine, Far Eastern Memorial Hospital, New Taipei City, Taiwan. ${ }^{4}$ Department of Chemical Engineering and Materials Science, Yuan-Ze University, Taoyuan, Taiwan. ${ }^{5}$ Departments of Laboratory Medicine, National Taiwan University Hospital, No. 7, Chung-Shan South Road, Taipei, Taiwan. ${ }^{6}$ Departments of Pediatrics, National Taiwan University Hospital, No. 7 , Chung-Shan South Road, Taipei, Taiwan.

Received: 12 June 2014 Accepted: 21 August 2014

Published online: 05 September 2014

\section{References}

1. Decramer M, Janssens W, Miravitlles M: Chronic obstructive pulmonary disease. Lancet 2012, 379:1341-1351.

2. Mathers $C D$, Loncar D: Projections of global mortality and burden of disease from 2002 to 2030 p38 MAP kinase inhibitors: a future therapy for inflammatory diseases. PLoS Med 2006, 3:e442.

3. Tuder RM, Petrache I: Pathogenesis of chronic obstructive pulmonary disease. J Clin Invest 2012, 122:2749-2755.

4. Barnes PJ: Mediators of chronic obstructive pulmonary disease. Pharmacol Rev 2004, 56:515-548.

5. Owen CA: Roles for proteinases in the pathogenesis of chronic obstructive pulmonary disease. Int I Chron Obstruct Pulmon Dis 2008 3:253-268.

6. Agusti A, Sobradillo P, Celli B: Addressing the complexity of chronic obstructive pulmonary disease: from phenotypes and biomarkers to scale-free networks, systems biology, and P4 medicine. Am J Respir Crit Care Med 2011, 183:1129-1137.

7. Fischer BM, Pavlisko E, Voynow JA: Pathogenic triad in COPD: oxidative stress, protease-anti-protease imbalance, and inflammation. Int I Chron Obstruct Pulmon Dis 2011, 6:413-421.

8. Roghanian A, Sallenave JM: Neutrophil elastase (NE) and NE inhibitors: canonical and non-canonical functions in lung chronic inflammatory diseases (cystic fibrosis and chronic obstructive pulmonary disease). J Aerosol Med Pulm Drug Deliv 2008, 21:125-144.

9. Lagente V, Le Quement C, Boichot E: Macrophage metalloelastase (MMP-12) as a target for inflammatory respiratory diseases. Expert Opin Ther Targets 2009, 13:287-295.

10. Shapiro SD, Goldstein NM, Houghton AM, Kobayashi DK, Kelley D, Belaaouaj A: Neutrophil elastase contributes to cigarette smoke-induced emphysema in mice. Am J Pathol 2003, 163:2329-2335.

11. Hogg JC, Chu F, Utokaparch S, Woods R, Elliott WM, Buzatu L, Cherniack RM, Rogers RM, Sciurba FC, Coxson HO, Paré PD: The nature of small-airway obstruction in chronic obstructive pulmonary disease. N Engl J Med 2004, 350:2645-2653.

12. Abboud RT, Vimalanathan S: Pathogenesis of COPD. Part I. The role of protease-anti-protease imbalance in emphysema. Int I Tuberc Lung Dis 2008, 12:361-367.

13. Korkmaz B, Horwitz MS, Jenne DE, Gauthier F: Neutrophil elastase, proteinase 3 , and cathepsin $\mathrm{G}$ as therapeutic targets in human diseases. Pharmacol Rev 2010, 62:726-759.

14. Reid PT, Marsden ME, Cunningham GA, Haslett C, Sallenave JM: Human neutrophil elastase regulates the expression and secretion of elafin (elastase-specific inhibitor) in type II alveolar epithelial cells. FEBS Lett 1999, 457:33-37.

15. Devaney JM, Greene CM, Taggart CC, Carroll TP, O'Neill SJ, McElvaney NG: Neutrophil elastase up-regulates interleukin-8 via toll-like receptor 4. FEBS Lett 2003, 544:129-132.

16. Fischer BM, Cuellar JG, Diehl ML, de Freytas AM, Zhang J, Carraway KL, Voynow JA: Neutrophil elastase increases MUC4 expression in normal human bronchial epithelial cells. Am J Physiol Lung Cell Mol Physiol 2003, 284:L671-L679.

17. Shao MX, Nadel JA: Neutrophil elastase induces MUC5AC mucin production in human airway epithelial cells via a cascade involving protein kinase $C$, reactive oxygen species, and TNF-a-converting Enzyme. $\mathrm{J}$ Immunol 2005, 175:4009-4016.

18. Park JA, He F, Martin LD, Li Y, Chorley BN, Adler KB: Human neutrophi elastase induces hyper-secretion of mucin from well-differentiated human bronchial epithelial cells in vitro via a protein kinase C-mediated mechanism. Am J Pathol 2005, 167:651-661.

19. Suzuki T, Moraes TJ, Vachon E, Ginzberg HH, Huang TT, Matthay MA, Hollenberg MD, Marshall J, McCulloch CA, Abreu MT, Chow CW, Downey GP: Proteinase-activated receptor-1 mediates elastase-induced apoptosis of human lung epithelial cells. Am J Respir Cell Mol Biol 2005, 33:231-247.

20. Nakajoh M, Fukushima T, Suzuki T, Yamaya M, Nakayama K, Sekizawa K, Sasaki H: Retinoic acid inhibits elastase-induced injury in human lung epithelial cell lines. Am J Respir Cell Mol Biol 2003, 28:296-304.

21. Sharafkhaneh A, Hanania NA, Kim V: Pathogenesis of emphysema: from the bench to the bedside. Proc Am Thorac Soc 2008, 5:475-477.

22. Tsao PN, Su YN, Li H, Huang PH, Chien CT, Lai YL, Lee CN, Chen CA, Cheng WF, Wei SC, Yu CJ, Hsieh FJ, Hsu SM: Over-expression of placenta growth factor contributes to the pathogenesis of pulmonary emphysema. Am J Respir Crit Care Med 2004, 169:505-511.

23. De Falco $\mathrm{S}$ : The discovery of placenta growth factor and its biological activity. Exp Mol Med 2012, 44:1-9.

24. Dewerchin M, Carmeliet P: PIGF: a multi-tasking cytokine with diseaserestricted activity. Cold Spring Harb Perspect Med 2012, 2:. doi:10.1101/ cshperspect.a011056.

25. DiPalma T, Tucci M, Russo G, Maglione D, Lago $C T$, Romano A, Saccone $S$, Della Valle G, De Gregorio L, Dragani TA, Viglietto G, Persico MG: The placenta growth factor gene of the mouse. Mamm Genome 1996, 7:6-12

26. Cheng SL, Wang HC, Yu CJ, Yang PC: Increased expression of placenta growth factor in COPD. Thorax 2008, 63:500-506.

27. Hou HH, Cheng SL, Liu HT, Yang FZ, Wang HC, Yu CJ: Elastase induced lung epithelial cell apoptosis and emphysema through placenta growth factor. Cell Death Dis 2013, 4:e793.

28. Janoff A, Sloan B, Weinbaum G, Damiano V, Sandhaus RA, Elias J, Kimbel P: Experimental emphysema induced with purified human neutrophil elastase: tissue localization of the instilled protease. Am Rev Respir Dis 1977, 115:461-478.

29. Cheng SL, Wang HC, Yu CJ, Tsao PN, Carmeliet P, Cheng SJ, Yang PC: Prevention of elastase-induced emphysema in placenta growth factor knock-out mice. Respir Res 2009, 10:115.

30. Lomas-Neira JL, Chung CS, Wesche DE, Perl M, Ayala A: In vivo gene silencing (with siRNA) of pulmonary expression of MIP-2 versus $K C$ results in divergent effects on hemorrhage-induced, neutrophil-mediated septic acute lung injury. J Leukoc Biol 2005, 77:846-853.

31. Nishimoto F, Sakata M, Minekawa R, Okamoto Y, Miyake A, Isobe A, Yamamoto T, Takeda T, Ishida E, Sawada K, Morishige K, Kimura T: Metal transcription factor-1 is involved in hypoxia-dependent regulation of placenta growth factor in trophoblast-derived cells. Endocrinology 2009, 150:1801-1808.

32. Green CJ, Lichtlen P, Huynh NT, Yanovsky M, Laderoute KR, Schaffner W, Murphy BJ: Placenta growth factor gene expression is induced by 
hypoxia in fibroblasts: a central role for metal transcription factor-1. Cancer Res 2001, 61:2696-2703.

33. Krämer B, Meichle A, Hensel G, Charnay P, Krönke M: Characterization of an Krox-24/Egr-1-responsive element in the human tumor necrosis factor promoter. Biochim Biophys Acta 1994, 1219:413-421.

34. Mura M, dos Santos CC, Stewart D, Liu M: Vascular endothelial growth factor and related molecules in acute lung injury. J Appl Physiol 2004, 97:1605-1617.

35. Hou HH, Cheng SL, Chung KP, Kuo YP, Yeh CC, Chang BE, Lu HH, Wang HC, Yu CJ: Elastase induces lung epithelial cells autophagy through placenta growth factor: a new insight of emphysema pathogenesis. Autophagy. In press.

36. Virolle T, Adamson ED, Baron V, Birle D, Mercola D, Mustelin T, de Belle I: The Egr-1 transcription factor directly activates PTEN during irradiation-induced signaling. Nat Cell Biol 2001, 3:1124-1128.

37. Salah Z, Maoz M, Pizov G, Bar-Shavit R: Transcriptional regulation of human protease-activated receptor 1: a role for the early growth response-1 protein in prostate cancer. Cancer Res 2007, 67:9835-9843.

38. Chen ZH, Kim HP, Sciurba FC, Lee SJ, Feghali-Bostwick C, Stolz DB, Dhir R, Landreneau RJ, Schuchert MJ, Yousem SA, Nakahira K, Pilewski JM, Lee JS, Zhang Y, Ryter SW, Choi AM: Egr-1 regulates autophagy in cigarette smoke-induced chronic obstructive pulmonary disease. PLoS One 2008, 3:e3316.

39. Thiel G, Cibelli G: Regulation of life and death by the zinc finger transcription factor Egr-1. J Cell Physiol 2002, 193:287-292.

40. Ngiam N, Post M, Kavanagh BP: Early growth response factor-1 in acute lung injury. Am J Physiol Lung Cell Mol Physiol 2007, 293:L1089-L1091.

41. Sakashita A, Nishimura Y, Nishiuma T, Takenaka K, Kobayashi K, Kotani Y, Yokoyama M: Neutrophil elastase inhibitor (sivelestat) attenuates subsequent ventilator-induced lung injury in mice. Eur J Pharmacol 2007, 571:62-71.

42. Bobic S, Seys S, De Vooght V, Callebaut I, Hox V, Dooms C, Vinckier S, Jonckx B, Saint-Remy JM, Stassen JM, Bullens DM, Ceuppens JL, Carmeliet P, Hellings PW: Placental growth factor contributes to bronchial neutrophilic inflammation and edema in allergic asthma. Am J Respir Cell Mol Biol 2012, 46:781-789.

43. Carter RI, Ungurs MJ, Mumford RA, Stockley RA: Aa-Val360: a marker of neutrophil elastase and COPD disease activity. Eur Respir J 2013, 41:31-38.

44. Adcock IM, Caramori G: Kinase Targets and inhibitors for the treatment of airway inflammatory diseases. BioDrugs 2004, 18:167-180.

45. Banerjee A, Koziol-White C, Panettieri R Jr: p38 MAPK inhibitors, IKK2 inhibitors, and TNFa inhibitors in COPD. Curr Opin Pharmacol 2012, 12:287-292.

46. Dempsey EC, Cool CD, Littler CM: Lung disease and PKCs. Pharmacol Res 2007, 55:545-559.

doi:10.1186/s12931-014-0106-1

Cite this article as: Hou et al:: PIGF mediates neutrophil elastase-induced airway epithelial cell apoptosis and emphysema. Respiratory Research 2014 15:106.

\section{Submit your next manuscript to BioMed Central and take full advantage of:}

- Convenient online submission

- Thorough peer review

- No space constraints or color figure charges

- Immediate publication on acceptance

- Inclusion in PubMed, CAS, Scopus and Google Scholar

- Research which is freely available for redistribution 